\title{
A High Performance Low Magnetic Field Internal Electrospray Ionization-Fourier Transform Ion Cyclotron Resonance Mass Spectrometer
}

\author{
Qinyuan Wu, Gordon A. Anderson, Harold R. Udseth, \\ Michael G. Sherman, Steven Van Orden, Ruidan Chen, \\ Steven A. Hofstadler, Michael V. Gorshkov, Dale W. Mitchell, \\ Alan L. Rockwood, and Richard D. Smith \\ Environmental Molecular Sciences Laboratory, Pacific Northwest National Laboratory, \\ Richland, Washington
}

\begin{abstract}
A new in-magnetic field electrospray ionization (ESI) and Fourier transform ion cyclotron resonance mass spectrometer has been constructed and evaluated. This system is characterized by the use of multiple concentric cryopanels to achieve ultrahigh vacuum in the ion cyclotron resonance cell region, a probe-mounted internal ESI source, and a novel in-field shutter. Initial experiments demonstrate high resolution mass measurement capability at a field strength of $1 \mathrm{~T}$. Mass resolution of 700,000 has been obtained for the $3+$ charge state of Met-Lys-bradykinin (at $m / z 440$ ) generated by electrospray ionization. When electron impact ionization was employed, resolution in excess of 9,200,000 was achieved for nitrogen molecular ions $\left(\mathrm{N}_{2}^{+}\right)$. Isotopic resolution for molecular ions of bovine ubiquitin (MW $=8565$

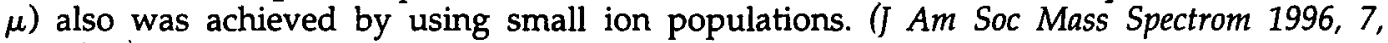
915-922)
\end{abstract}

$\mathrm{T}$ The coupling of electrospray ionization (ESI) with Fourier transform ion cyclotron resonance mass spectrometry (FTICR-MS) [1, 2] has been shown to be a powerful analytical tool [3-5]. Due to the multiple charging phenomenon inherent to the ESI process, ions are typically generated in a mass-tocharge ratio range accessible to most mass analyzers. The presence of multiple charge states can make spectral interpretation more challenging, particularly for the analysis of mixtures. Therefore, a high resolution mass spectrometer is beneficial to the use of ESI. Fourier transform ion cyclotron resonance (FTICR) has been shown to be capable of providing ultrahigh resolution [6, 7], ion remeasurement [8-10], and multistage mass spectrometry (MS ${ }^{n}$, where $\left.n>2\right)$ [11]. Molecular ions in the mega-Dalton mass regime recently have been transferred nondestructively into the gas phase by using ESI and, due to the high number of charges on each ion, individual ions have been isolated and studied by using FTICR $[12,13]$. MS ${ }^{4}$ measurements have been performed on multiply charged protein and oligonucleotide ions by using ESI-FTICR [14, 15]. ESI-

Address reprint requests to Dr. Richard D. Smith, Environmental Molecular Sciences Laboratory, Pacific Northwest National Laboratories, Battelle Boulevard, P8-19, P.O. Box 999, Richland, WA 99352.
FIICR has been used to study several types of weakly bound noncovalent biomolecular complexes that include DNA-drug [16], protein-protein [17], and DNA-protein complexes [18]. A number of recently reported novel techniques for ion manipulation based on quadrupolar axialization have provided improved performance [19] and enhanced dynamic range [20, 21].

The conventional approach for coupling ESI with FTICR involves formation of ions outside the magnetic field and injection of these ions through the fringing magnetic field by using either radiofrequency ion guides [22] or electrostatic lenses [23]. Significant ion losses can occur during ion transport from an external ESI source [3, 5, 24]. In an attempt to increase detection sensitivity, Hofstadler and Laude [25] moved the electrospray source into the high field region of a 3-T magnet and obtained the required pressure reduction by employing differentially pumped concentric vacuum chambers. It was demonstrated that significantly higher ion currents could be transmitted to an ion cyclotron resonance (ICR) cell than by typical external ESI source designs. However, such a design imposes a strict requirement on the differential pumping system because ESI occurs at atmospheric pressure and high resolution FTICR detection requires ultrahigh vacuum. 
Ion detection in the initial work was performed at $\sim 10^{-7}$ torr, which resulted in low mass resolution spectra. Subsequent work that employed the high magnetic field ESI source in conjunction with the concentric vacuum chambers approach has demonstrated improved performance with the addition of a mechanical shutter to enhance differential pumping [26].

Here we describe the design and performance of a novel in-field ESI-FTICR instrument in which adverse pressure effects are substantially overcome with enhanced differential pumping. The instrument includes a movable (in $x-y-z$ directions) ESI probe, five stages of differential pumping, and a new in-field shutter. Of particular significance is that high performance results have been obtained at much lower magnetic field strength ( $1 \mathrm{~T}$ ) than typically employed (3-7 T) for ESI-FTICR, which suggests the potential for the design of high resolution FTICR instrumentation by using permanent magnets.

\section{Instrumentation}

Figure 1 shows a schematic of the instrumentation. The entire vacuum system and the associated components are cart-mounted to enable easy and rapid movement of the vacuum chamber into the bore of the magnet. For enhanced vibrational isolation, the pumping system is located on a separate side cart that is moved in tandem with the main vacuum system cart, and the instrument can be moved in or out of the magnet while ultrahigh vacuum (UHV) is maintained. ICR cell control and data collection cables are connected from the opposite side of the magnet. The bore of the magnet has a $10 \frac{3}{4}$-in. diameter (with a room temperature shim system). The magnet (Oxford Instruments, Ox- ford, England; maximum field of $4.7 \mathrm{~T}$ ) was charged to $1 \mathrm{~T}$ for the present experiments (to confine the 5-G line of the fringing magnetic field in the present location, the magnet operation is restricted to a field strength of $1 \mathrm{~T})$.

\section{Vacuum System}

The vacuum system incorporates five stages of differential pumping that provide approximately 12 orders of magnitude decrease in pressure between the ESI source and the ICR, a distance of about 9 in. The main vacuum chamber is a titanium nitrite coated aluminum tube of 10-in. o.d. and 9.75-in. i.d. (Spectra Physics, Mountain View, CA). Inside this primary vacuum chamber is a set of five concentric tubes $(\sim 40$-in. length and 1/16-in. thickness) with a spacing of $1 / 4$ in. between any two adjacent tubes. The materials of these tubes are (in order of descending diameters) aluminum, copper, aluminum, copper, and aluminum. The aluminum tubes function as radiation shields, whereas the copper tubes are "cryopanels" (see subsequent text). The first two tubes (aluminum and copper) serve to pump the fifth vacuum stage and surround the entire ICR cell region (see Figure 1), whereas the inner three tubes (aluminum, copper, and aluminum) provide the pumping for the fourth stage. These two stages are separated by a 2-mm-diameter conductance limit (electrically isolated from the chamber) mounted on the third aluminum tube, and are cooled separately by two cryopumps (model RGD 1245 coldhead from Leybold Vacuum Products, Inc., Export, PA). The copper panels and the aluminum shields are connected to the inner $(10-20 \mathrm{~K})$ and outer stages $(40-100 \mathrm{~K})$ of the two coldheads, respectively. The coldhead housing is

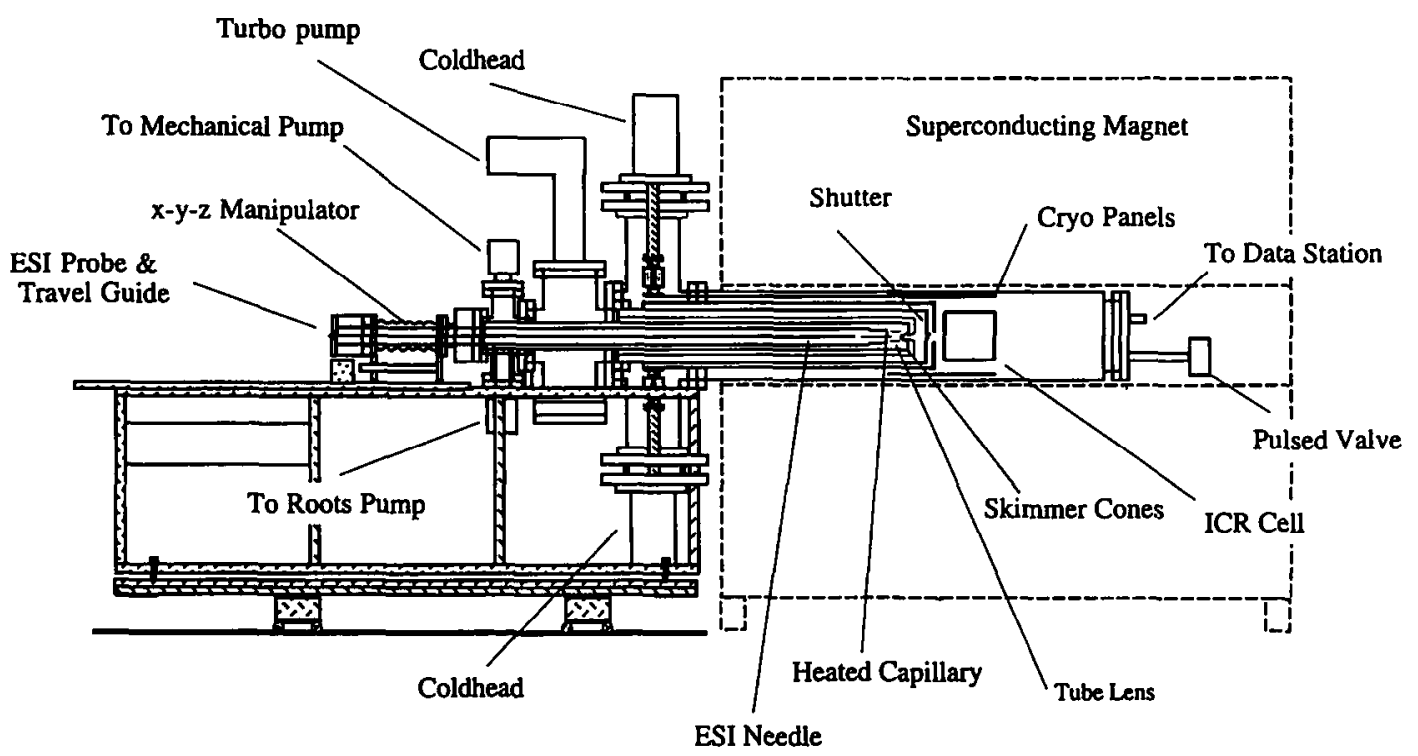

Figure 1. A schematic of the in-field electrospray ionization Fourier transform ion cyclotron resonance mass spectrometer (ESI-FTICR/MS). The 4.7-T superconducting magnet was used at a field strength of $1-T$ (see text). The instrument includes a movable (in $x-y-z$ directions) ESI probe, five stages of differential pumping, and a new in-field shutter design, and obtains UHV conditions at the cell appropriate for high performance detection. 
flange-mounted to the main chamber through a vibration-isolation bellows, which significantly reduces low frequency vibrational noise in the detected spectra. In addition to the cryopanel, the fifth stage chamber also is pumped by a turbomolecular pump (model 5400, $400 \mathrm{~L} / \mathrm{s}$, Alcatel Vacuum Products). The pressure at this stage is measured by using an ion gauge mounted on the same cross as the turbomolecular pump and the cryopumps. Cernox ${ }^{\mathrm{TM}}$ temperature sensors (Lakeshore Measurement and Control Technologies, Westerville, $\mathrm{OH})$ are attached to the end (i.e., near the ICR cell) of each of these cryopanels and cryoshields.

The entire set of cryopanels is supported by (but thermally isolated from) a 6-in.-o.d. aluminum tube in the third stage chamber, on which is mounted a novel in-field shutter assembly (see subsequent text) and an electrically isolated plate that has a 3-mm-diameter aperture. The third stage is pumped by a turbomolecular pump (Alcatel model 5400). Inside the third stage chamber are two concentric tubes of 4- and 3-in.-o.d. that serve as the second and the first stage vacuum regions, respectively. Mounted on the second stage tube is a skimmer with a 1.8-mm-diameter orifice (model 2, Beam Dynamics, Minneapolis, MN) that is positioned $2 \mathrm{~mm}$ ("tip to tip") from another skimmer on the first stage tube (3-in. o.d.). The first stage skimmer (homemade) has a $0.6-\mathrm{mm}$-diameter orifice and a $90^{\circ}$ cone. An electrospray probe (vide post) can be inserted into the first stage chamber. A mechanical pump (model 2063, $30 \mathrm{~L} / \mathrm{s}$, Alcatel Vacuum Products, Hingham, MA) is used to pump the second stage region between the two skimmers and a roots blower pump (model WSU 251, displacement $179 \mathrm{ft}^{3} / \mathrm{min}$, Leybold) is used to pump the first stage chamber.

The cryopanels in the fifth and the fourth stage normally obtain a temperature of $\sim 15 \mathrm{~K}$, which results in a fifth stage base pressure of $\sim 8 \times 10^{-10}$ torr. During operation in the ESI mode, pressures are estimated (based on the gauge readings when the chamber is removed from the magnet bore, and the calculated conductance for the various stages) to be 1-3 torr, $10^{-2}$ torr, $10^{-5}$ torr, $10^{-7}$ torr and $10^{-9}-10^{-10}$ torr, in the first through fifth stages, respectively (see Figure 2).

\section{Electrospray Ionization Probe and Source Assembly}

To minimize vacuum chamber contamination and allow easy access to the electrospray source elements, a probe-mounted electrospray ionization source was developed that comprised a tube lens, a heated metal capillary inlet, a sheath gas and countercurrent gas system, and an electrospray emitter (see Figure 2). To maximize the conductance of the various pumping stages, the diameter of the probe tube was limited to 2 in. (o.d.), which imposed considerable constraints upon mechanical design. The probe is mounted on a motor driven linear travel guide, which serves to move the probe into or out of the chamber. A double O-ring

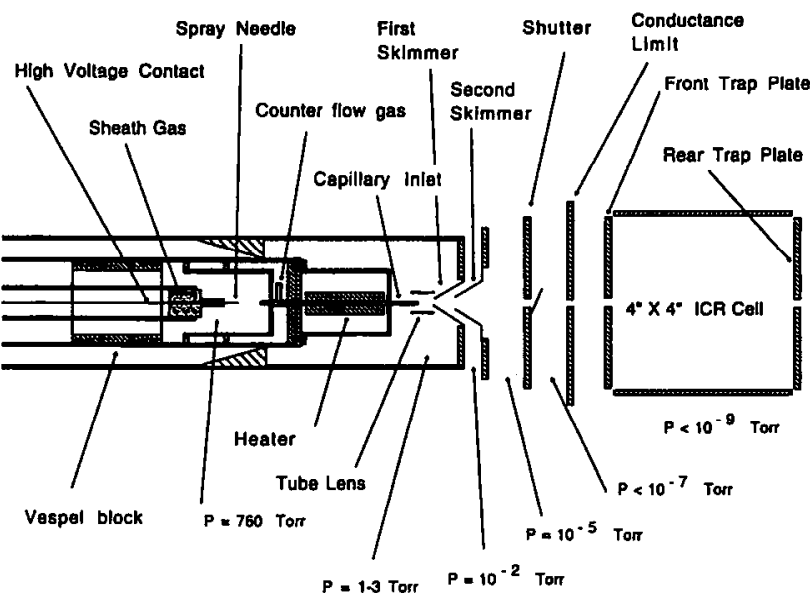

Figure 2. Detailed illustration of the beam optics for the instrumentation and the estimated pressures at various stages (see text).

arrangement is used to maintain the vacuum seal between the probe tube and an $x-y-z$ motion manipulator (MDC Vacuum Products Corp., Hayward, CA) on the first stage chamber. A gate valve is used to close the inlet system when the ESI probe is removed, so that the first and the second stage chambers can be isolated from their respective pumping systems. Thereforc, there is no need to vent the vacuum chamber to replace or clean the ESI source assembly or the heated capillary inlet. After the ESI probe is inserted into the chamber, it is disconnected from the travel guide, and the position of the tube lens and the heated capillary inlet then can be optimized with respect to the first skimmer by adjusting the $x-y-z$ manipulator. Whereas the probe is $\sim 85 \mathrm{in}$. long, and its mechanical stability at the capillary end was found to effect the ESI performance greatly, four Teflon pieces were installed inside the second stage tube to provide additional support for the probe (see Figure 2).

All of the materials used for the ESI probe are nonmagnetic. The temperature on the heated capillary is controlled by an automatic feedback circuit (Spectrum Solutions, Inc., Russelton, PA), and a high voltage-low current heating scheme is used. The capillary is heated indirectly by three cartridge heaters inserted into a copper block that houses about two-thirds of the 5.5-in.-long stainless steel capillary $(1 / 16$-in. o.d., 600 $\mu \mathrm{m}$ i.d.). This design not only facilitates capillary replacement, but also eliminates the need to electrically float the power supply and the temperature controller for the heated capillary inlet. No significant noise was detected by the ICR detection circuitry from this capillary control unit. The tube lens was fabricated from a $70 \%$ transmitting screen $(7 \mathrm{~mm}$ long $\times 1 \mathrm{~cm}$ diameter) that extends $\sim 4 \mathrm{~mm}$ beyond the heated capillary (see Figure 2). The electrospray source capillary (emitter) was stainless steel with an i.d. of about $100 \mu \mathrm{m}$ (200$\mu \mathrm{m}$ o.d.) and is connected to a $100-\mu \mathrm{m}-\mathrm{i} . \mathrm{d}$. by $\sim 70-$ in.-long fused silica sample inlet capillary (sample dead 
volume $\approx 14 \mu \mathrm{L}$ ). Both the spray emitter and the sample inlet capillary are installed in a supporting tubing assembly that also includes the high voltage lead. The high voltage can be applied directly when a stainless steel ESI emitter is used or indirectly when a fused silica capillary (e.g., a "microspray" emitter) is used (see Figure 2) [27]. Samples were injected by using a syringe pump (Harvard Apparatus, South Natick, MA). The ESI tubing assembly slides into another aluminum tube, which can be moved in the $x-y$ direction with respect to the heated inlet capillary; the $z$ direction (i.e., parallel to the magnetic field lines) can be adjusted by moving the emitter assembly.

\section{Shutter}

To minimize the effects of the higher pressure ESI source and differential pumping stages on ion manipulation and detection processes in the FTICR cell, a shutter assembly was developed based upon the motion of a current loop in a magnetic field (Kouzes, $R$. T.; Peurrung, A. J., private communication). As illustrated in Figure 3, the shutter consists of a rotatable plate ( 1 in. $\times 9 / 16$ in. $\times 1 / 8$ in.) and a $45^{\circ}$ aluminum pole ( 1 in. $\times 1 / 8$-in. diameter) around which insulated coils were wrapped ( $\sim 400$ turns). When a direct current $(i)$ is passed through the coil, as indicated in Figure 3, a magnetic dipole moment $(\mu)$ is induced,

$$
\mu=N i A
$$

where $N$ is the number of turns of the coil and $A$ is the loop area of the coil [28]. The interaction of this dipole moment with the magnetic field (B, the boldface letters represent vectors) imposes a torque $(\tau)$ on the current loop because

$$
\tau=\mu \times \mathrm{B}
$$

This results in a force (F) sufficient to open the shutter,

$$
\tau=\mathbf{r} \times \mathbf{F}
$$

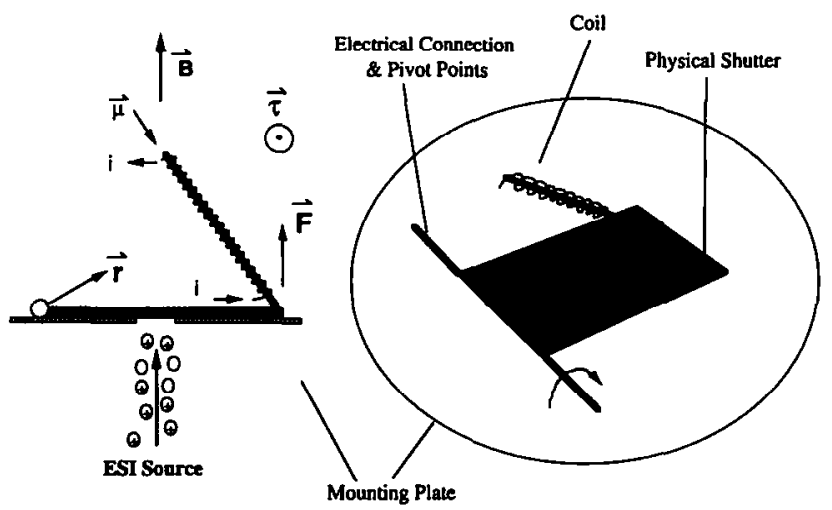

Figure 3. The design and principle of the in-field shutter developed in this work (see text for the definitions of the symbols used). The shutter can rotate to open or closed position by passage of a direct current through the coil. in which $\mathbf{r}$ is the vector from the pivot point to the center of mass of the shutter. Note that the opening force changes as the shutter rotates with respect to the magnetic field direction. Similarly, the shutter can be closed by reversing the current applied. Experimentally, friction is introduced on the pivot points by using thin copper shims as electrical contacts, which ensures good electrical conductivity and prevents the shutter from bouncing back to the open (or closed) position. The shutter opening time is controlled by a TTL pulse from a data station (Finnigan FTMS, Madison, WI) through a specially designed control circuit; provision also was made to open or close the shutter manually. Typically, a 2.5-A current is needed to open or close the shutter. The key advantage of this design is that current is applied only during the opening or closing of the shutter, which eliminates a potentially excessive heat load on the shutter coils and the introduction of magnetic field perturbations during excitation/detection events.

\section{Ion Cyclotron Resonance Cell}

The ICR cell (a 4-in. cubic design) was mounted on a spring-loaded frame attached to the rear flange (10-in. diameter) of the main vacuum chamber. There are four copper rods on the fourth stage aluminum shield chamber (on which the "conductance limit" is mounted; see Figure 2) that slide into four holes on the self-adjustable frame, which automatically aligns the ICR cell with the ion beam from the ESI source. Ions enter the ICR cell through a 6-mm-diameter aperture in the center of the front trap plate. An electron impact ionization (EI) source was located near the rear plate of the cell to allow the electron beam to be injected into the cell through a 2-mm-diameter hole on the rear trap plate. The EI source consisted of a filament, a collector, and an electron gate. All of the cell and EI source control cables were connected to the data station through the rear flange. For the experiments reported here, a homemade preamplifier was used [29]. A quadrupolar excitation circuit with a dipole-quadrupole switching device (constructed in our laboratory) also was incorporated. Broadband quadrupole cooling [30] and selective ion accumulation [20,21] waveforms were generated by using an arbitrary waveform generator (PCIP-arbitrary waveform generator, $5 \mathrm{MHz}, 12$ bit, Keithley MetraByte Co., Taunton, MA) controlled via a PC. Both the waveform output and the circuit switching were controlled by the data station. A piezoelectric pulsed valve (Lasertechnics, Inc., Albuquerque, NM) was mounted on the rear flange (see Figure 2) and controlled by TTL pulse from the data station. Dry $\mathrm{N}_{2}$ gas was pulsed into the chamber through this valve and transported to the ICR cell region through an aluminum tube (1-in. i.d.) that terminated $\sim 1$ in. from the rear trap plate. 


\section{Experimental Conditions}

In a typical ESI-FTICR experiment, samples were injected at $0.3-0.5 \mu \mathrm{L} / \mathrm{min}$ by using a syringe pump. All samples were obtained from commercial sources (Sigma Chemical Co., St. Louis, MO) and were dissolved in a $5 \%$ HOAc solution that contained $50 \%$ $\mathrm{MeOH}$. For the experiments reported here, typical potentials on the electrospray emitter, heated capillary, tube lens, first skimmer, second skimmer, shutter, and conductance limit are $3-3.5 \mathrm{kV}, 20-35 \mathrm{~V}, 215-220 \mathrm{~V}$, $7-10 \mathrm{~V}, 2.5-5 \mathrm{~V},-6-10 \mathrm{~V}$, and $0 \mathrm{~V}$, respectively. The temperature of the capillary inlet typically was maintained at $\sim 100{ }^{\circ} \mathrm{C}$ for aqueous samples. An ion current of $30-40 \mathrm{pA}$ (which likely includes contributions from ions that are not completely desolvated) normally was detected by a picoampere meter (model 617, Keithley Instruments, Inc., Cleveland, $\mathrm{OH}$ ) at the shutter (closed) for the Met-Lys-bradykinin sample ( 0.4 $\mathrm{mg} / \mathrm{mL}$ ), of which up to $10 \mathrm{pA}$ of ion current was measured (when the shutter was open) at the rear trapping plate of the ICR cell (with the front trapping plate grounded). The kinetic energy of the ions was $7 \pm 0.5 \mathrm{eV} / \mathrm{q}$ when the foregoing settings, as measured by biasing the front trap plate (i.e., retarding field methud), were employed. No significant ion current was detected at the ICR cell when the system was withdrawn from the magnetic field under otherwise identical conditions, which indicates that there is at least a tenfold increase in ion transmission in the 1-T magnetic field. Based on previous reports at 3-T field strength [25], the ion current to the ICR cell is expected to further increase when the magnetic field strength is increased to $4.7 \mathrm{~T}$.

A typical ESI mass spectrometry experimental sequence included ion quenching (1 s), injection (opening the shutter) and trapping (by the pulsed introduction of $\mathrm{N}_{2}$ gas), ion "cooling" (with the shutter closed), dipolar excitation, and detection steps. Ion injection time varied from $50 \mathrm{~ms}$ to a few seconds (depending on the ion current), and cooling times were 5-10 s. During the ion accumulation period, a constant $1-\mathrm{V}$ difference was maintained on the front and the rear trap plates to facilitate accumulated trapping [31]. In an electron impact experiment, the injection event was replaced by an ionization step in which the potential on the electron gate was lowed to admit electrons into the cell and to ionize the gas molecules introduced through the pulsed valve.

\section{Results and Discussion}

\section{Electrospray Ionization Source Performance}

The ESI source was designed so that the potentials and the relative positions of the beam optics could be varied, which allowed experiments to be conducted to study the effects of various electrostatic configurations on the ion intensity, ion kinetic energy, ion trapping efficiency, and ion transmission discrimination. Several such experiments have been performed to date. Similar to the previous studies with single skimmer design [25], ion kinetic energy (measured by the retarding field method) was found to increase linearly from 4 to $10 \mathrm{eV} / \mathrm{q}$ as the direct current potential on the first skimmer was varied from 3 to $18 \mathrm{~V}$. Lower skimmer potentials largely decreased the accuracy of the kinetic energy measurement because the ion current typically decreased to below $3 \mathrm{pA}$. A nonzero intercept of $\sim 2.5$ $\mathrm{eV} / \mathrm{q}$ in a plot of ion kinetic energy versus the first skimmer potential may result from the voltage applied on the second skimmer $(2.5 \mathrm{~V})$ in these experiments. However, some contribution from molecular beam slip, as has been shown for a single skimmer interface [32], also is likely.

Similar to a previous single skimmer design [25], ion current was found to be affected significantly by the distance between the tube lens and the first skimmer. As the skimmer-tube lens distance decreases from 1.8 to $0.1 \mathrm{~cm}$, the ion current measured on the rear trap plate increases steadily until it reaches a plateau at a distance of $<2.5 \mathrm{~mm}$. It was observed also that the pressure in the second and the third stages increased as the tube lens and heated capillary inlet were moved closer to the skimmer. For normal operation, this capillary-skimmer distance was set at 1.0-1.5 mm. Another factor that had a major effect on the ion current was the tube lens, but in a very different way. We found that by adding a tube lens immediately adjacent to the heated capillary, the ion current is much less dependent upon the relative position of the capillary and the skimmer in the $x-y$ directions, which greatly enhances normal operation of the ESI source. By using the foregoing experimental conditions, the highest ion signal was obtained when the tube lens biased at $210 \pm 15 \mathrm{~V}$. Our experience to date indicates that in its present configuration, ESI described source is convenient and reliable despite its internal location and lack of provision for visual inspection (as is typical for external source configurations).

\section{Ion Injection and Trapping}

In agreement with previous studies [25], the ion kinetic energy was found to depend greatly on the ESI source parameters, and ions of different mass-to-charge ratios could have different kinetic energies. In one typical ESI study, the kinetic energy of the $2+$ charge state ions (at $m / z$ 662) of Met-Lys-bradykinin was $\sim 7-8 \mathrm{eV} / \mathrm{q}$, whereas that of the $3+$ charge state ions (at $\mathrm{m} / \mathrm{z} 441$ ) was $\sim 9-10 \mathrm{eV} / \mathrm{q}$. Consequently, a potential difference of $1 \mathrm{~V}$ between the front and the rear trap plates (i.e., the trapping "window") during ion injection was observed to produce the greatest total ion intensities (e.g., the sum of $2+$ and $3+$ intensities for Met-Lys-bradykinin). Narrower (than $1 \mathrm{~V}$ ) trapping 
windows decreased the trapping efficiency. A wider window, on the other hand, allowed some ions to escape the trapping field because ions only lose a limited amount of their kinetic energy during transit between the two trap plates and, even if reflected, would exit the trap if their kinetic energy was still sufficient to exceed the barrier provided by the front trap plate potential [33].

Shutter performance was examined by using the optimized conditions described in the preceding text. Under conditions below the space-charge limit of the ICR cell, the total ion intensity for Met-Lys-bradykinin increased linearly with the length of the shutter-open interval. For most experiments the shutter-open interval was typically $0.5 \mathrm{~s}$ or longer. The shutter effectively controls ion injection for events as short as $30 \mathrm{~ms}$ (the width of the TTL pulse). Shorter shutter-open experiments are limited at present, because they require reversing the current flow direction in a much shorter time (and the practical constraints on the power supply circuit used). The practical shortest "open" time feasible with this design arises from the maximum current possible and the moment of inertia to be overcome.

To demonstrate the effectiveness of the shutter, Figure 4a shows an expanded spectrum of the $2+$ charge state ions of Met-Lys-bradykinin with a transient (longer than $1 \mathrm{~s}$ ) obtained with the normal experimental procedure. The $y$ scale is relative to the $3+$ charge state peak, which is the most intense component. When the shutter was kept open for about $2 \mathrm{~min}$ and remained open through the entire excitation-detection events under otherwise identical conditions as for Figure $4 a$, the length of the transient was shortened and spectral resolution was degraded significantly, as illustrated in Figure 4b. Similar transient length and slightly better resolution (than in Figure $4 \mathrm{~b}$ ) were obtained when a $10-\mathrm{V}$ potential was applied to the conductance limit to prevent the ions from entering the ICR cell except during the ion injection period (Figure 4c). Similar effects also were observed for the $3+$ charge state ions, except that the extents of frequency drift was not as large as in the case of the $2+$ charge state ions (most likely owing to its much larger ion population). Evidently, the small ESI ion current, in addition to the neutral beam, significantly degraded the ion excitation and/or detection performance. The decrease in peak intensities in the open-shutter experiments was due to the shorter transient lifetime and the frequency drift, which mainly resulted from ion-ion interactions, as can be seen by comparing the spectra in Figure $4 \mathrm{~b}$ and $4 \mathrm{c}$. Similar effects also were observed for other species.

\section{Initial Results}

Initial experiments that utilized electron impact ionization yielded a resolution of $9,200,000$ [full width at half
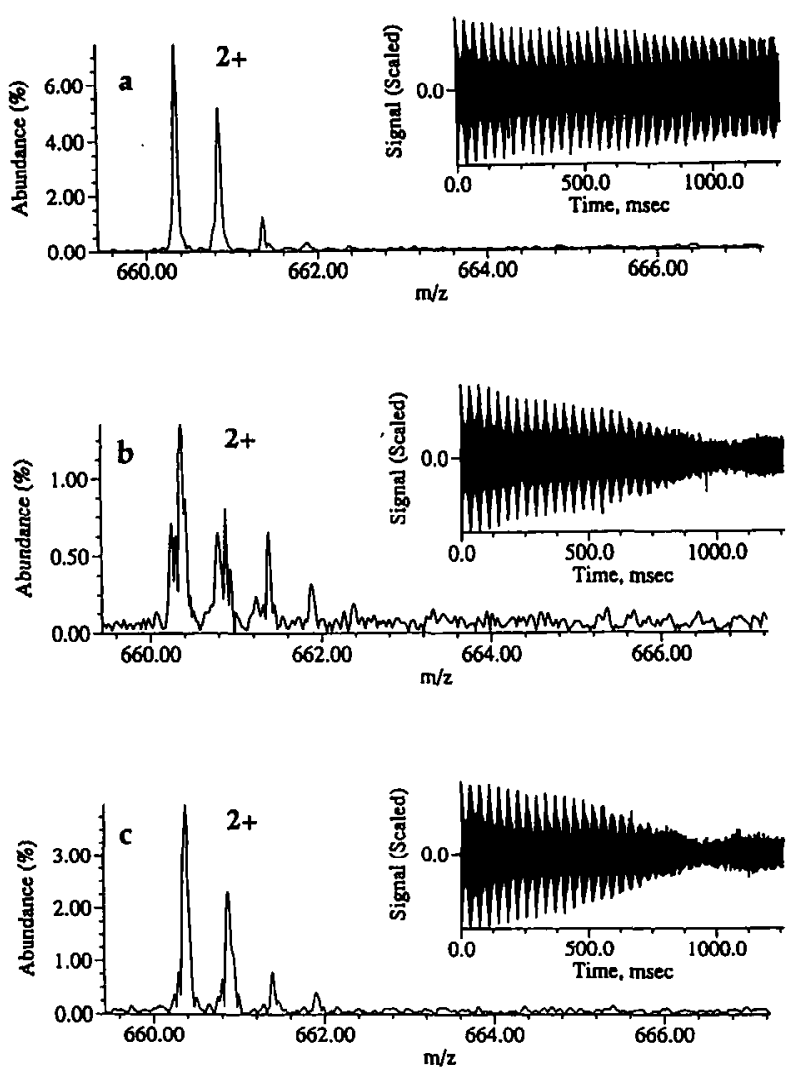

Figure 4. The effects of the shutter on transient lifetime and spectrum resolution for Met-Lys-bradykinin: (a) Typical ESI mass spectrum and time domain signal for the 2 + charge state ions, obtained when the shutter was open only during the ion injection event. (b) Spectrum and transient obtained $2 \mathrm{~min}$ after the shutter was opened and held open through the entire experimental sequence. (c) Spectrum and transient for the same conditions as in (b), except the lens potentials were adjusted to prevent ions from entering the cell during cooling, excitation, and detection (see text).

maximum (FWHM)] for $\mathrm{N}_{2}^{+}$ions, with transient lifetime in excess of $40 \mathrm{~s}$ and signal-to-noise ratio of 600 . In the negative ion mode, $\mathrm{SF}_{6}^{-} \mathrm{EI}$ spectra were obtained with transient lengths exceeding $3 \mathrm{~min}$. The peak width [FWHM in real-mode fast Fourier transform (FFT)] of the $\mathrm{N}_{2}^{+}$ions was only $35 \mathrm{mHz}$, which is comparable with a value of $25 \mathrm{mHz}$ for $\mathrm{O}^{+}$ions $\left(m / \Delta m<3 \times 10^{8}\right)$ obtained under a much lower base pressure of $<10^{-12}$ torr in a 7-T ICR [34]. Similar performance also has been demonstrated for ${ }^{4} \mathrm{He}^{+}$ ions detected in a 7-T ICR with a resolution of $2 \times 10^{8}$ [35]. This shows that the ICR cell, low pressure detection environment, and magnetic field homogeneity are conducive to acquisition of high performance mass measurements, even at a magnetic field strength of $1 \mathrm{~T}$.

Figure 5 shows spectra from electrospray ionization of various proteins and peptides: (a) Met-Lysbradykinin ( $M W=1320$ ), (b) melittin ( $M W=2845$ ua), and (c) bovine ubiquitin ( $\mathrm{MW}=8565 \mathrm{u}$ ). Each of these spectra was obtained from a single experimental se- 

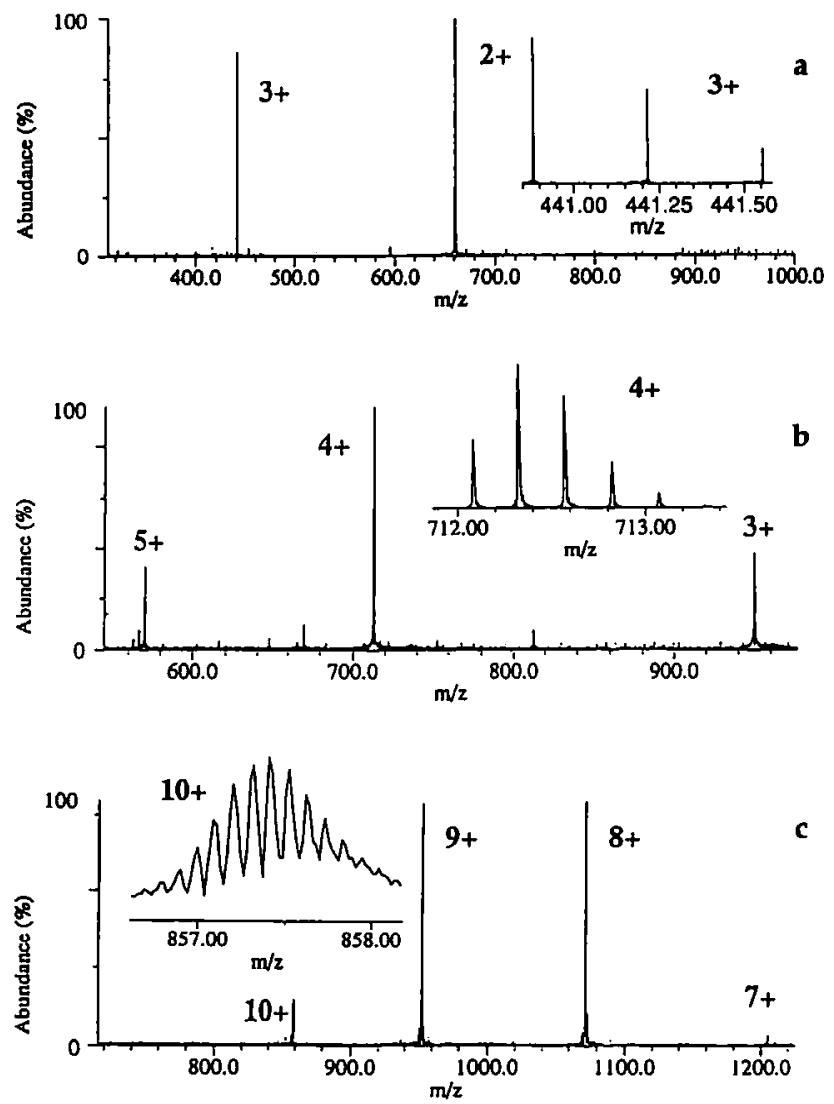

Figure 5. Initial electrospray ionization spectra. (a) Met-Lysbradykinin ( $\mathrm{MW}=1320 \mathrm{u}$ ) and the isotopic pattern of the $3+$ charge state peak (the inset at $m / z$ 440) with a resolution (FWHM) of 700,000. (b) Melittin (MW $=2845 \mathrm{u}$ ) and a spectrum resolution of 70,000 (FWHM at $\mathrm{m} / z$ 712). (c) Ubiquitin (MW = $8565 \mathrm{u}$ ). The $10+$ charge state of ubiquitin was resolved isotopically (the inset) with $<50 \%$ of the $10+$ charge state ions in (c). Each spectrum is the result of one experimental sequence [except the inset in (c), which was the sum of 50 experiments].

quence (except for the inset of Figure 5c, which is the sum of 50 experimental sequences) by using $40-60-V_{p-p}$ chirp excitation $(100 \mathrm{~Hz} / \mu \mathrm{s}$, from $\mathrm{m} / \mathrm{z} 200$ to 2000$)$ and broadband detection with 512,000 data points. The spectra were subjected to a triangle apodization and one zero filling before FFT. The inset of Figure 5a shows isotopic detail for the $3+$ charge state peak of Met-Lys-bradykinin, which demonstrates a resolution (FWHM) of 700,000 at $\mathrm{m} / z 440$ (transient length $>40$ s). The inset in Figure 5b shows the resolved isotopic distribution for the $4+$ charge state of melittin and a spectrum resolution of 70,000 (FWHM).

However, under conditions employed for melittin, isotopic resolution was not obtained for ubiquitin (Figure 5c) until $>50 \%$ of the initial ion population for the $10+$ charge state was ejected by large amplitude $\left(>30 \mathrm{~V}_{\mathrm{p}-\mathrm{p}}\right.$ ) off-resonance dipolar excitation for $500 \mathrm{~ms}$ in the presence of $\mathrm{N}_{2}$ gas $\left(\sim 10^{-7}\right.$ torr), after which the isotopic resolution was observed clearly. Similar results also were obtained by simply injecting a smaller population of ions (Figure 5c inset). A small ion popu- lation was also needed to give a resolved utopic pattern for cytochrome $c(12,360 \mathrm{Da})$. It has been shown both theoretically and experimentally [36-38] that ions with similar mass-to-charge ratios, such as those that differ isotopically, can become "locked" in the same cyclotron frequency (i.e., "phase locking") when the number of such ions in an ICR cell exceeds a certain limit. This limit has been predicted to be proportional to the square of the magnetic field strength, the ion cyclotron radius and inversely proportional to the square of mass of the ion, among other factors [36]. In the case of ubiquitin, this limit can be estimated to be about 3000 ions in the $10+$ charge state at 1-T field strength, in qualitative agreement with the foregoing observation.

\section{Conclusions}

A new in-magnetic field electrospray ionization (ESI) Fourier transform ion cyclotron resonance mass spectrometer (FTICR-MS) has been constructed and its initial performance has been evaluated. This new system is characterized by its unique ultrahigh vacuum capability in the cell region that is obtained by using a dual concentric cryopanel arrangement, a probe-mounted ESI source, and a novel in-(magnetic) field shutter. We have shown that the in-field shutter improves the system performance by blocking the entrance of both the ions and the neutrals from the ESI source to the ICR cell in events other than during ion injection-trapping (and causing both frequency drifting and shorter transient lifetimes). Initial spectra demonstrate that high resolution mass measurements are obtainable even at magnetic field strength of only $1 \mathrm{~T}$. Mass resolution (FWHM) of $9,200,000$ for $\mathrm{N}_{2}^{+}$ions generated from electron impact ionization and of 700,000 (transient lifetime $>40 \mathrm{~s}$ ) for the $3+$ charge state ions of Met-Lys-bradykinin (at $m / z$ 440) from electrospray ionization were obtained. Isotopic resolution for molecular ions of bovine ubiquitin ( $M W=8565 \mathrm{u}$ ) also was achieved, but required limiting the ion population. Substantially higher resolving power and dynamic range (and high sensitivity) are anticipated when the magnetic field is increased to its maximum $(4.7 \mathrm{~T})$. The experimental results shown here indicate that, with certain technical improvements, high performance might be obtainable for FTICR with other types of magnets, for example, permanent magnet or electromagnets, and at field strength lower than $1 \mathrm{~T}$.

\section{Acknowledgments}

The authors thank Dr. R. T. Kouzes and Dr. A. J. Peurrung for the initial contributions to the shutter design, J. Follansbee for construction of the shutter controller, and B. Higgins and H. Phillips for mechanical work. This work was supported by the U.S. Department of Energy under Contract DE-AC06-76RLO 1830. Pacific Northwest National Laboratory is operated by Battelle Memorial Institute for the U.S. Department of Energy. 


\section{References}

1. Marshall, A. G.; Grosshans, P. B. Anal. Chem. 1991, 63, A215-A229.

2. Comisarow, M. B.; Marshall, A. G. Chem. Phys. Lett. 1974, 25, 282-283.

3. Henry, K. D.; Williams, E. R.; Wang, B. H.; McLafferty, F. W.; Shabanowitz, J.; Hunt, D. F. Proc. Natl. Acad. Sci. USA 1989, $86,9075-9078$.

4. Hofstadler, S. A.; Laude, D. A. Anal. Chem. 1992, 64, 569-572.

5. Winger, B. E.; Hofstadler, S. A.; Bruce, J. E.; Udseth, H. R.; Smith, R. D. J. Am. Soc. Mass Spectrom. 1993, 4, 566-577.

6. Bruce, J. E.; Anderson, G. A.; Hofstadler, S. A.; Winger, B. E.; Smith, R. D. Rapid Commun. Mass Spectrom. 1993, 7, 700-703.

7. Beu, S. C.; Senko, M. W.; Quinn, J. P.; McLafferty, F. W. J. Am. Soc. Mass Spectrom. 1993, 4, 190-192.

8. Guan, Z. Q.; Hofstadler, S. A.; Laude, D. A. Anal. Chem. 1993, 65, 1588-1593.

9. Williams, E. R.; Henry, K. D.; McLafferty, J. W. J. Am. Chem. Soc. 1990, 112, 6157-6162.

10. Speir, J. P.; Gorman, G. S.; Pitsenberger, C. C.; Turner, C. A.; Wang, P. P.; Amster, I. J. Anal. Chem. 1993, 65, 1746-1752.

11. Freiser, B. S. Talanta 1985, 32, 697-708.

12. a. Bruce, J. E.; Cheng, X.; Bakhtiar, R.; Wu, Q.; Hofstadler, S. A.; Anderson, G. A.; Smith, R. D. J. Am. Chem. Soc. 1994, 116, 7839-7847. b. Smith, R. D.; Cheng, X.; Bruce, J. E.; Hofstadler, S. A.; Anderson, G. A. Nature 1994, 369, 137-139.

13. Cheng, X.; Camp, D. G.; Wu, Q.; Bakhtiar, R.; Springer, D. L.; Morris, B. J.; Bruce, J. E.; Anderson, G. A.; Edmonds, C. G.; Smith, R. D. Nucl. Acid Res. 1996 in press.

14. Senko, M. W.; Beu, S. C.; McLafferty, F. W. Anal. Chem. 1994, 66, 415-417.

15. Wu, Q.; Van Orden, S.; Cheng, X.; Bakhtiar, R.; Smith, R. D. Anal. Chem. 1995, 67, 2498-2509.

16. Cheng, X.; Chen, R.; Bruce, J. E.; Schwartz, B. L.; Anderson, G. A.; Hofstadler, S. A.; Gale, D. C.; Smith, R. D.; Gao, J.; Sigal, G. B.; Mammen, M.; Whitesides, G. M. J. Amer. Chem. Soc. 1995, 117, 8859-8860.

17. Bruce, J. E.; Van Orden, S. L.; Anderson, G. A.; Hofstadler, S. A.; Sherman, M. G.; Rockwood, A. L.; Smith, R. D. J. Mass Spectrom. 1995, 30, 124-133.

18. Cheng, X.; Harms, A. C.; Goudreau, P. N.; Terwilliger, T. C.; Smith, R. D. Proc. Natl. Acad. Sci. 1995 in press.
19. Schweikhard, L.; Guan, S. H.; Marshall, A. G. Int. J. Mass Spectrom. Ion Processes 1992, 120, 71-83.

20. Bruce, J. E.; Anderson, G. A.; Hofstadler, S. A.; Van Orden, S. L.; Sherman, M. S.; Rockwood, A. L.; Smith, R. D. Rapid Commun. Mass Spectrom. 1993, 7, 914-919.

21. Bruce, J. E.; Anderson, G. A.; Smith, R. D. Anal. Chem. 1996, $68,534-541$.

22. McIver, R. T. J.; Hunter, R. L.; Bowers, W. Int. I. Mass Spectrom. Ion Processes 1985, 64, 67-77.

23. Kofel, P.; Allemann, M.; Kellerhails, H. Int. J. Mass Spectrom. Ion Processes 1985, 65, 97-103.

24. Henry, K. D.; Quinn, J. P.; McLafferty, F. W. I. Am. Chem. Soc. 1991, 113, 5447-5449.

25. Hofstadler, S. A.; Laude, D. A. J. Am. Soc. Mass Spectrom. 1992, 3, 615-623.

26. Guan, Z. Q.; Campbell, V. L.; Drader, J. J.; Hendrickson, C. L.; Laude, D. A. Rev. Sci. Instrum. 1995, 66, 4507-4515.

27. Gale, D. C.; Smith, R. D. Rapid Commun. Mass Spectrom. 1993, 7, 1017-1021.

28. Halliday, D.; Resnick, R. Physics, 4th ed. Wiley: New York, 1962.

29. Anderson, G. A.; Bruce, J. E.; Hofstadler, S. A.; Rockwood, A.; Smith, R. D. Proceedings of the 43rd ASMS Conference on Mass Spectrometry and Allied Topics; Atlanta, GA, May 1995.

30. Guan, S. H.; Wahl, M. C.; Marshall, A. G. J. Chem. Phys. 1994, 100, 6137-6140.

31. Beu, S. C.; Laude, D. A. Int. J. Mass Spectrom. Ion Processes 1991, 104, 109-127.

32. Campbell, V. L.; Guan, Z. Q.; Laude, D. A. J. Am. Soc. Mass Spectrom. 1994, 5, 221-229.

33. Hofstadler, S.; Wu, Q.; Bruce, J. E.; Chen, R.; Smith, R. D. Int. J. Mass Spectrom. Ion Processes 1995, 142, 143-150.

34. Bamberg, M.; Allenmann, M.; Wanczek, K. P. Proceedings of the 35th ASMS Conference on Mass Spectrometry and Allied Topics; Denver, CO, May 1987; pp 1116-1117.

35. Alber, G. M.; Marshall, A. G.; Hill, N. C.; Schweikhard, L.; Ricca, T. L. Rev. Sci. Instrum. 1993, 64, 1845-1852.

36. Mitchell, D. W.; Smith, R. D. Phys. Rev. E 1995, 52, 4366-4386.

37. Huang, J. Y.; Tiedemann, P. W.; Land, D. P.; McIver, R. T.; Hemminger, J. C. Int. J. Mass Spectrom. Ion Processes 1994, $134,11 \cdot 21$.

38. Naito, Y.; Inoue, M. J. Mass Spectrom. Soc. Japan 1994, 42, 1. 